\title{
EL BALANCE DE LA INDEPENDENCIA LATINOAMERICANA
}

\author{
ÁDÁM ANDERLE
}

Universidad de Szeged

\section{The gains and losses of Latin American independence}

This study is a historiographical overview of the literature of the Latin American wars of independence. It analyses the gains and losses, and poses the question: "has the world advanced" in the 200 years of independence? The first part of the article concentrates on the events of the wars of independence and the developments in the 19th century focusing on the works of Francisco Morales Padrón, Luis Navarro Garia, José Carlos Mariategui, and the approach of the German historian Manfred Kossok. In the second part the author presents the question of subdesarrollo and dependencia. He discusses the different interpretations for insufficient progress from the positivist viewpoints to the assessment of the economists of the CEPAL. The novelyy of this part is that it presents the results of the comparative analyses (Wittman, Paich, Zimányi) published in Hungarian bistoriography in the 1960s-1970s that revealed the similarities between the progress in Central-Eastern Eurape and Latin America.

La revisión de la historiografía sobre las interpretaciones de la historia de los 200 años de la independencia y de la guerra de independencia latinoamericana es una tarea desesperante - ni los estudios hechos para la conmemoración del bicentenario (inicio de la guerra de la independencia 1810) pueden ofrecer un cuadro completo. Debido al creciente número de publicaciones, el investigador debe apoyarse cada vez más en sus propias lecturas y experiencias personales historiográficas. La historiografía del subcontinente, por supuesto, es una creación colectiva de la comunidad internacional de investigadores ${ }^{1}$, en la cual los resultados/fracasos de estos 200 años son temas permanentes del balance de la independencia. "¿Hemos avanzado o no?" - he aquí una pregunta que sigue estando de actualidad en América Latina.

\section{La historiografia de la guerra de independencia latinoamericana}

El tema está lleno de interpretaciones contradictorias.

Los primeros estudios latinoamericanos describieron la guerra como la lucha de la sociedad rural criolla (blanca) contra los centros urbanos coloniales.

\footnotetext{
1 Vid. Francois CHEVALIER, América Latina de la independencia a nuestros dias. Barcelona, Ed. Labor, 1983; Manuel LUCENA SALMORAL (coord.), Historia de Iberaamérica. Tomo III., Madrid, Ed. Cátedra, 1988; Nelson MARTÍNEZ DÍAZ, La Independencia Hispanoamericana. Madrid, Col. Historia $16,1999$.
} 
En la época del romanticismo, las historias oficiales de cada país latinoamericano llamaron béroes y libertadores a quienes, bajo influencia de la guerra de independencia norteamericana y de la revolución francesa, lucharon contra los españoles opresores. Según esta visión, que sigue presente en los himnos nacionales hasta hoy, los patriotas son jóvenes, guapos y buenos, y los realistas son viejos, feos, incultos y malos.

En la historiografia española, los estereotipos fueron diferentes durante un siglo y medio: los americanos eran traidores perversos, rebeldes... y se alzaron por influencia de Inglaterra y de los masones ${ }^{2}$. Entre las críticas a personajes de la época sobresale la que hizo Marx sobre uno de los protagonistas de la guerra de la independencia ${ }^{3}$, Simón Bolivar, de quien provee una imagen extremadamente distorsionada, casi caricaturesca.

La opinión negativa sobre las guerras de independencia fue muy común y fuerte también en la época de Franco. Las jóvenes repúblicas latinoamericanas -según la propaganda franquista- fueron "anárquicas e inestables desde su nacimiento, y se convirtieron lógicamente en anarquía y semidemocracias". La prensa española del siglo $\mathrm{XX}$ utilizó semejante tono. Iberoamérica es "un continente español; el cual fue mutilado por la independencia", dijeron ${ }^{4}$.

El libro de Francisco Morales Padrón, sobre la historia de América, publicado en 1975, año de la muerte de Franco, ya muestra un cambio en el pensamiento español. Según Morales Padrón, el pueblo español reaccionó "con perplejidad e indiferencia" ante los sucesos iberoamericanos: "la lucha en el propio suelo peninsular contra los franceses -en alusión a la invasión de Napoleón en 1808- restó la atención a lo que sucedió en Ultramar", escribe Morales Padrón, luego continúa: "el silencio que se hace en la prensa y la Gaceta es asombroso."

Silencio, indiferencia, aceptación son las palabras claves del comportamiento de la época, resume Morales Padrón ${ }^{5}$.

Sin embargo, más tarde, durante el siglo XIX vieron la luz otras interpretaciones sobre la guerra de independencia. En la opinión general, fue una simple guerra civil entre los criollos y los peninsulares. Esta percepción subraya como características singulares la sintonía incréble de las regiones y los fuertes sentimientos regionales entre los combatientes ${ }^{6}$.

En el siglo XX nacieron también estudios ya basados en la investigación científica y en las fuentes archivísticas. Los más interesados en examinar los antecedentes de la guerra de independencia, así como la crisis del absolutismo ilustrado en la Península

2 Luis NAVARRO GARCÍA, "La Independencia de Hispanoamérica", in: Balance de la bistoriografia sabre Iberoamérica (1945-1988). Pamplona, Ediciones de Universidad de Navarra, 1989, 531-532. Ver también LUCENA SALMORAL op.cit., 21-23.

${ }^{3}$ MARX, "Bolivar y Ponte". Marx-Engels Müvei, 14., Budapest, Kossuth, 167-180.

4 Monserrat HUGUET SANTOS, La difusión de la imágen de Amética Latina en la prensa española del primer franquismo"; Rosa Maria PARDO SANZ, "Percepciones y decisiones en la política iberoamericana del primer franquismo", in: Monserrat HUGUET SANTOS (ed.), La formación de la imagen de América Latina en España, 1898-1989. Madrid, Espasa-Calpe, 1992, 78., 348.

5 Francisco MORALES PADRÓN, Historia General de América. Madrid, Ed. OEI, 1975, 131-132.

${ }^{6}$ N. MARTÍNEZ DÍAZ, op.cit., 9-12, Lucena Salmoral op.cit., 25-26. 
Ibérica, fueron los historiadores ingleses y españoles. Hoy ya es obvio que al final del siğlo XVIII el Imperio Español colonialista estaba por completo en desintegración. Ello se refleja en las reformas planificadas para América por Godoy, Aranda y Francisco de Miranda, que intentan buscar alternativas futuras para el Imperio Español.

El análisis historiográfico más completo sobre este tema fue realizado por Luis Navarro García, quien hizo una reseña sobre la guerra de independencia para la conferencia internacional de historiadores organizada en 1988 en Navarra. Navarro García tomó en cuenta tanto las causas internas como las externas. Las causas internas, según él, fueron las desigualdades sociales internas existentes, la decadencia de la economía española y la política económica despótica de la madre patria, que quería utilizar las fuentes económicas provenientes de sus colonias para su propia modernización, lo cual generó resistencia.

Entre las causas externas, menciona la influencia de la Ilustración francesa, y, más tarde, de las ideas liberales, la guerra de independencia exitosa de las colonias inglesas norteamericanas, la revolución francesa, la invasión napoleónica de España y la divulgación del liberalismo español en las colonias?.

Aparece como una tendencia historiográfica independiente la comparaicón de las dos guerras de independencia americanas - la inglesa en el Norte y la española en el Sur. Esta comparación, por supuesto, presta más atención a las exterioridades: a los hechos de que las guerras de las colonias españolas fueron más largas, que cometieron muchas atrocidades, destrucciones y causaron más víctimas mortales. Llama la atención también el raquítico papel que jugó la comunidad internacional en estos conflictos. Morales Padrón.provee datos sobre las pérdidas: la población disminuyó un $50 \%$ en Venezuela y Uruguay debido a los muertos, la migración y las huidas y las enfermedades. Los resultados también son diferentes en las dos Américas, dice. Mientras las colonias españolas se convirtieron en varios estados independientes, en América del Norte se puede observar un proceso de unificación de las colonias inglesas ${ }^{8}$.

Esta tendencia/intención comparativa está presente hasta hoy en la historiografia universal, concretándose al final en la investigación de la revolución. Según ésta, las luchas por la independencia latinoamericana pertenecen a la época de las revoluciones burguesas (Hobsbawm). En la línea de investigación de Manfred Kossok, de Leipzig, se habla de un ciclo americano en esta época revolucionaria que se inició con la guerra de independencia norteamericana de las colonias inglesas, seguida por la revolución de Haití y luego por la guerra de independencia latinoamericana $(1810-1824)^{9}$. En mi

7 NAVARRO GARCÍA, op.cit., 529-555. Ver Tibor WITTMAN, Latin-Amerika története. Budapest, 1978, Gondolat, 203. Id., "A spanyol gyarmatok függetlenségi háborújának jellege az újabb történeti irodalomban", in: Acta Historica, XXVIII. Szeged, 1968, 15-28.

8 F. MORALES PADRÓN, op. cit., 139; Eloy NAVARRO DOMINGUEZ - Antonio GUTIÉRREZ ESCOBEDO, "La independencia hispanoamericana: predicciones y precursores", in: Orbis Incognitus. Avisos y Legajos del Nuevo Mundo. Huelva, Universidad de Huelva, 2007, 269-275.

9 Manfred KOSSOK, La revolución en la bistoria de América Latina, La Habana, Ed. Ciencias Sociales, 1989, 155-178; Semejante tono utiliza Sergio GUERRA VILABOY, Historia y revolución en América Latina, La Habana, 1989. La última obra de este autor, Jugar con fuego, guerra social y 
opinión, la rebelión peruana de Túpac Amaru (1780-1783) pertenece también a este ciclo, señalando el preludio de la guerra de independencia contra los españoles.

Los historiadores marxistas evitaron la discusión de este problema hasta finales de los años 1950 debido a la biografía muy negativa y llena de prejuicios que escribió Marx sobre Bolívar - respetando el "canon" marxista"

El carácter y contenido de la guerra de independencia fue un tema aparte, discutible especialmente entre los historiadores marxistas. ¿Fueron tevoluciones (también) o "sólo" guerras de independencia? O ¿tal vez contenían en si dicha dualidad...?

La participación de J. C. Mariátegui en este debate fue temprana. Él habla sobre una "revolución de la independencia" en su libro 7 ensayos de interpretación de la realidad peruana (1927). Según él, los procesos latinoamericanos fueron determinados por las necesidades del desarrollo capitalista de la civilización occidental. No obstante, según Mariátegui, los elementos mínimos del capitalismo estaban presentes sólo en las colonias de La Plata, es decir, en la costa atlántica. La región andina sufría el feudalidad, los terratenientes criollos y la servidumbre.

Por eso es que en América Latina, dice Mariátegui, "la revolución ganó por la solidaridad continental de los pueblos". Según Mariátegui, en estas condiciones nació el continentalismo: "el nacionalismo continental de los revolucionarios hispanoamericanos se sumó a esa mancomunidad forzosa de sus destinos para nivelar a los pueblos más avanzados en marcha hacia el capitalismo con los más retrasados en la misma vía."11 En la interpretación de Mariátegui, la meta de- la guerra de la independencia era la eliminación del subdesarrollo y acercarse al mismo nivel de desarrollo que los países del "Primer Mundo".

Hoy ya se considera común y corriente la opinión de Mariátegui, la cual evalúa los cambios ocurridos entre "las nubes de la política" (Marx) como una revolución politica, mientras que en la realidad social siguieron en vigencia la servidumbre y la feudalidad. En resumen, la discontinuidad y la continuidad están presentes al mismo tiempo en las guerras de independencia, mientras que la eliminación de la esclavitud, la apropiación de las tierras eclesiásticas coloniales y la creación de las constituciones liberales pusieron en marcha importantes cambios sociopolíticos.

El alemán Manfred Kossok (Leipzig) tuvo un argumento interesante a favor del carácter revolucionario burgués de las guerras de independencia: "En Sudamérica dominaba el factor militar como fuerza motriz de la revolución." De tal manera, continúa Kossok "el ejército tuvo que compensar la extrema debilidad del elemento burgués..."12.

utopia en la guerra de independencia, ganó el premio del Bicentenario de la Casa de las Américas en 2010. Ed. ADHULAC, La Habana, 2011.

10 Ádám ANDERLE, Vihar a Sierrában, Budapest, Gondolat, 1982.

${ }^{11}$ José Carlos MARIÁTEGUI, Siete ensayos de interpretación de la realidad peruana, Lima, Minerva, 1972, 17,62 .

12 Manfred KOSSOK, op. cit, 278. 
Sin embargo, Morales Padrón opina que el balance de las luchas por la independencia no es negativo: "Socialmente, a raiz de la lucha se originó una gran movilidad social. ( ..) la situación de algunas de ellas (clases) mejoró y hubo cierta apertura social." Pero, continúa, "los indios y negros, la capa más baja de la sociedad, no sufrieron tantas transformaciones como la clase intermedia $(\cdots)$ Toda la sociedad sufrió cambios en sus modos, maneras y costumbres. A causa de la guerra se cerraron muchas escuelas y universidades, se multiplicaron los cafés, se acentuó la tendencia al lujo, los desastres de la contienda fueros un incentivo para los médicos extranjeros. Se realizaron muchas obras públicas. En resumen, todos los cambios del siglo XIX su vislumbran o apuntan ya en menor escala dentro de la etapa de la independencia" 13 .

Por supuesto, esta opinión es demasiado positiva, y Mariátegui, el ya mencionado pensador peruano, muestra también el lado sombrío de la situación. Según él, durante el siglo $\mathrm{XX}$ "se inauguró así un régimen que cualesquiera que fuesen sus principios, empeoraba en cierto grado la condición de los indígenas en vez de mejorarla". La revolución no dio poder así no más a una clase. Tampoco eliminó la servidumbre, porque la revolución no tocó al latifundio ${ }^{14}$. Esta línea de pensamiento formó luego la base de la poco transparente interpretación "semifeudal" que apareció en el diccionario soviético-marxista en el siglo XX.

Luis Vitale, en cambio, escribe sobre el carácter burgués de la revolución de 1810, considerándolo una creación de la burguesía criolla. Pertenecen a esta: los hacendados, los comerciantes, los tabacaleros, los ganaderos costeños, los dueños de las minas, los latifundistas que producen la yerba mate - todos los que están en contacto con el mercado capitalista internacional en formación; también, los intelectuales que están relacionados con ellos, especialmente los juristas. Vitale utiliza ejemplos de Argentina, Chile, Paraguay y Uruguay, pero André Gunder Frank usa los mismos ejemplos para generalizar, y subraya el carácter burgués/capitalista de la guerra de independencia, que apareció como una revolución burguesa y depositó el poder en manos de la burguesía ${ }^{15}$.

No obstante, Tibor Wittman opina que esta guerra fue "el primer movimiento exitoso y de inmenso poder del mundo colonial...". Wittman, aunque la pone en el ciclo de las revoluciones burguesas, no le atribuye características "capitalistas": "las guerras libertadoras nacionales comenzaron con América Latina", constata ${ }^{16}$. Las revoluciones burguesas que estallaron en los centros capitalistas vinieron acompañadas por las guerras libertadoras en la periferia (colonias) ${ }^{17}$. Estas guerras comenzaron en América Latina.

${ }^{13}$ F. MORALES PADRÓN, op. cit., 141.

${ }^{14} \mathrm{~J}$.C. MARIÁTEGUI, op. cit., 69.

15 André GUNDER FRANK está de acuerdo en su libro Lumpenburguesia, lumpendesarrollo. México, ERA, 1971, 56-61.

16 WTTTMAN, op. cit., 202.

${ }^{17}$ Estudié este problema en la época de las revoluciones tempranas. Vid. Ádám ANDERLE, "Forradalom a centrumban - felkelés a periférián? 1450-1650." Acta Historica, T. 91., 1990, 3-15.

El estudio fue publicado en español y en catalán. 


\section{Los problemas del subdesarrollo y de la dependencia}

A esta corriente de pensamiento corresponde el problema del subdesarrollo, que es el gran sentimiento latinoamericano del fin del siglo XIX y del inicio del XX, alimentado por los fracasados intentos de los "románticos" en los años 1850 y las políticas gubernamentales positivistas, que no lograron conseguir el resultado esperado ${ }^{18}$. Tal es el argumento que esgrimían los que buscaban las causas del atraso/subdesarrollo.

Hubo otras explicaciones. Una interpretación norteamericana encontró las causas del subdesarrollo en las raíces españolas de los criollos, utilizando principalmente los aspectos negativos de la "leyenda negra" sobre la mentalidad española ${ }^{19}$. Este pensamiento norteamericano sentó la base del Manifest Destiny, que legitimó la política y las intrusiones político-militares de los Estados Unidos en los países del subcontinente.

La política real de los Estados Unidos apareció dentro del panamericanismo desde finales del siglo XIX. Sin importar en qué práctica política concreta apareciera (big stick policy, diplomacia de dólares, "buena vecindad", relaciones interamericanas, Alianza para el Progreso) siempre utilizó la justificación de lo necesario de la modernización ${ }^{20}$.

El pensamiento político latinoamericano apunto como causa del subdesarrollo a la heterogeneidad de la población del continente y la existencia distintas razas en el período que va desde el inicio del siglo XIX hasta la crisis económica mundial del siglo XX (1929-1933). Bolivar ya se había enfrentado a este problema anteriormente, aunque él todavía hablaba de la creación de las normas de la democracia étnica/racial. No obstante, la clase dominante en los países nuevos criollos (blancos) vieron la causa del subdesarrollo en la piel de color. los grupos sociales de los indígenas, los negros, los mulatos y los mestizos; y la explicación principal era el color de la piel, las diferencias étnico-raciales. El primero en sugerir la asimilación de los indígenas y favorecer la migración europea fue tal vez el mexicano Francisco Pimental. El lema del argentino Alberdi "gobernar es poblar" sugiere la misma política, pero ya entre los marcos continentales. Estas ideas, aceptadas y llevadas a cabo por los gobiernos positivistas, tenían como meta el "blanqueo" y la mezcla de la sangre, lo cual había que reforzarse con políticas educativas apropiadas. Las interpretaciones y conceptos democráticos basados en la igualdad racial (Ezequiel Zamora, Benito Juárez, José Martí) no llegaron a ser una fuerza de gobierno en el siglo XIX.

Desde el fin de las últimas décadas del siglo XIX, con el progreso de la mezcla étnica/racial, el mestizaje y su conversión en un estrato social mayoritario en todos los países del continente, empezaron a surgir opiniones que relacionaban el futuro de

${ }^{18}$ Ádám ANDERLE, Nemzettudat és kontinentalizmus Latin-Amerikában a XIX. és a XX. sqázadban. Budapest, Kossuth, 1988, 77-92, 105-149.

19 Vid. Julian JUDERÍAS, La leyenda negra. Estudios acerca del concepto de España en el extranjero. Madrid, Ed. Nacional, 1974 (edición nueva, 2009); Ádám ANDERLE, "La leyenda negra en Hungría en los siglos 19 y 20", Trienio (Madrid), 1985, · 6, 134-160.

${ }^{20}$ Vid. Ádám ANDERLE, Latin-Amerika története. $2^{2}$ edición ampliada, Szeged, SZTE Juhász Gyula Tankönyvkiadó, 2010. 
América Latina con lo mestizo. El más conocido ejemplo tal vez lo constituye el libro La raza cósmica, de José Vasconcelos. Mientras tanto, Fernando Ortiz y Gilberto Freyre imaginaban la sociedad armónica como la síntesis de todas las razas y de los diferentes colores de la piel (cubanidad, brasilianidad).

No obstante, las políticas criollas, aun en su diversidad, siempre se basaban en el racismo y el color de la piel, y esperaban acercarse al nivel de los países desarrollados copiando (mimetismo) o adaptando sus métodos.

Sin embargo, estas expectativas de crecimiento no se cumplieron. El sentimiento de atraso se incrementó a fines del siglo XIX y comienzos del XX, dando lugar al sentimiento del subdesarrollo. Esto señaló ya tanto las diferencias cuantitativas del desarrollo latinoamericano como también las cualitativas (deformes). Por lo tanto, el sentimiento del subdesarrollo al inicio del siglo XX se alimentó de esta sensación, y puso la base para los programas de los partidos políticos emergentes que buscaban un terrer camino de desarrollo ${ }^{21}$.

La crisis económica mundial afectó profundamente a los países latinoamericanos, cuya base económica era la exportación, pero arrojó luz sobre el hecho de que la relación entre el subcontinente y la economía mundial era fuerte y asimétrica. La teoría de la dependencia estructural fue elaborada por Raúl Prebitsch. Utilizó el concepto centroperiferia para modelar la relación entre los países capitalistas desarrollados y América Latina. La periferia está subordinada al centro, al cual provee de agtomaterias primas baratas y con bajo valor adicional. Utilizándola como base, después de la Segunda Guerra Mundial los economistas de la Comisión Económica para América Latina de las Naciones Unidas (Commision for Latin America and the Carribean, CEPAL), Prebitsch, Celso Furtado, F. H. Cardoso, Aníbal Pinto y otros (Arturo Frondizi, Victor Urquidi) elaboraron el desarrollismo, la tesis del "fomento e industrialización", que proponía la industrialización interna como principio del fomento y el desarrollo y, por ende, la reducción de las importaciones en los países latinoamericanos.

La teoría de la dependencia pertenece a esta línea de pensamiento que dio una dimensión histórica al subdesarrollo (Cardoso, Gunder Frank, Aníbal Quijano y otros), y lo examinó en el sistema de relaciones asimétricas de la economía mundial (worldsystem)22 apenas en formación (Wallerstein).

Una variación temprana de esta la podemos observar ya en el pensamiento de Mariátegui. Según él, los capitalistas de Inglaterra, los banqueros de Londres, financiaron con créditos el nacimiento de las repúblicas. "Estos capitalistas, dice Mariátegui, como colonizadores de nuevo tipo"23, querían controlar los mercados latinoamericanos.

${ }^{21}$ Escribo detalladamente sobre el tema en mi libro "Nemqettudat és kontinentaližmus ...".

22 F. H. CARDOSO y E. FALETTO, Dependencia y desarrollo en América Latina. Ensayo de interpretación sociológica. México, Siglo Veintiuno, 1969; T. DOS SANTOS, "El nuevo carácter de 1a dependencia". Universidad de Chile, Cuaderno 10, 1968. Ver también Tamás SZENTES, Polgári és "ujibaloldali"' elméletek a tôkés világgazdaságról. Budapest, Kossuth, 1980.

${ }^{23}$ J. C. MARIÁTEGUI, op. cit., 67. 
El pensamiento anticolonialista en el siglo XX por lo tanto se conecta con las teorías de la dependencia.

Además de analizar esta dependencia externa de los países "centrales", André Gunder Frank dirigió su atención a los factores internos, subrayando el carácter lumpen de la burguesía local (lumpenburguesía), que le lleva a hablar de lumpendesarrollo. Los economistas que favorecen esta teoría sugieren diferentes caminos para la eliminación de la dependencia. Los partidarios del modelo desarrollo en dependenicia imaginan el desarrollo autónomo mediante un estado fuerte e intervencionista - como lo intentaron varios países populistas latinoamericanos (Argentina, Chile, Brasil, México). Por otro lado, los representantes de la nueva izquierda y radicales como el propio Gunder Frank, llegaron a ver la necesidad de una revolución socialista ${ }^{24}$.

Estas teorías (desarrollismo, teoria de las dependencias) tuvieron una fuerte influencia en el pensamiento de la economía política latinoamericana en los años 1950-70, y están de vuelta, después de las dictaduras militares y las décadas neoliberales, en el pensamiento del subcontinente - especialmente en la práctica de los gobiernos neopopulistas.

Analizando los resultados de las líneas de investigación de la latinoamericanística húngara, más bien, en un sentido más amplio, de la historiografía y las ciencias económicas húngaras, se observa que los investigadores húngaros en los años 1960-70 pertenecían a las corrientes principales de investigación internacionales, y que llegaron a conclusiones semejantes a las latinoamericanas.

Tibor Wittman en su libro Az árforradalom és a világpiaci kapisolatok kezdeti mozzanatai (La revolución de precios y la formación inicial del mercado mundial) (1957), ya muy tempranamente, mencionó la idea de la formación del mercado mundial -sistema mundial- observando el movimiento de los precios en el siglo XVI dentro del marco histórico universal. Lo interesante es que Wittman, y luego Vera Zimányi, ampliaron el estudio del movimiento de los precios en Hungría mirando el problema en coordenadas internacionales ${ }^{25}$.

En la historia económica húngara Zsigmond Pál Pach, gracias al estudio de los problemas de los siglos XVI-XVII, observó que los fenómenos de la división del trabajo entre Europa Oriental y Occidental eran consecuencia del reordenamiento de las vías comerciales internacionales centro-periferia. Así pudo demostrar que desde la segunda mitad del siglo XVII América Latina conectó con Europa Occidental en el nuevo mercado mundial (productos de las plantaciones), mientras que Europa Central y Oriental se desconectó de esta modema división del trabajo capitalista internacional ${ }^{26}$. Era obvio que la posición ocupada por Europa Central y Oriental y por América Latina en el sistema de

${ }^{24}$ A. GUNDER FRANK, Lumpenburguesía ..., 147-154.

25 Vera ZIMÁNYI, “A magyarországi ármozgások helye az európai fejlödésben”. Történelmi Szemle, 1972, 5. sz. 378-410.

${ }^{26}$ Zsigmond Pál PACH, "A nemzetközi kereskedelmi útvonalak átrendezôdésének kérdéséhez", in: Századok. 1968, 863-891. 
la economía mundial emergente era semejante -periférica. Los estudios regionales comparativos de Tibor Wittman demostraron las semejanzas en las características del agrodesarrollo en los siglos XVI-XVII: el latifundio conectado al mercado mundial cuya estructura interna conservó las instituciones de la servidumbre feudal ${ }^{27}$.

La investigación comparativa profunda de las dos regiones periféricas no tuvo continuación, principalmente debido a la muerte temprana de Wittman. Otra posible razón es las condiciones políticas de la época: América Latina fue clasificada como "tercermundista", Europa Central-Oriental, perteneciente al sistema socialista, como "segundomundista", y. esta clasificación creó perspectivas distintas sobre las regiones, e hizo indeseable la comparación.

Las investigaciones de Wittman, que se concentraron en los temas sui generis latinamericanos, respondiendo particularmente a las preguntas sobre el desarrollo andino y caribeño (plantaciones, minería, desarrollo estatal etc.) trajeron nuevos resultados.

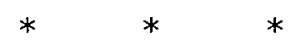

La analogia "América Latina-Europa Central" surgida en los estudios de Tibor Wittman en un aspecto histórico y metodológico, reapareció después del cambio del orden político en Hungría (1989-1990), aunque en otro aspecto. El concepto de la "latinoamericanización" primero apareció en la prensa -bajo una luz muy negativa: se refería a la confusión política, la brutalidad y el desempacho en el estilo político; todo lo que se llama politiqueria en América Latina, o "selva polititia" en Perú.

Durante el estudio de los partidos políticos húngaros nos hemos percatado del hecho de que el Foro Democrático Húngaro (MDF), entonces en formación, tenía las características de los partidos políticos populistas latinoamericanos en tanto que partido colectivo, "partido vertical". Mientras, los partidos Demócratas Libres (SzDSz) y Jóvenes Demócratas (FIDESZ) aparecían como partidos liberales europeos de estrato social, y el Partido Socialista Húngaro (MSzP) indeciso entre ser un partido europeo izquierdista $o$ un partido populista de base social amplia28.

Sin embargo, las tendencias de la transformación democrática anteriormente mencionadas parecen aludir a un proceso de "latinoamericanización". En 2010, el FIDESZ ganó las elecciones al estilo de un partido definitivamente populista, al tiempo que los partidos de tipo "europeo" (el también centrista MDF) se debilitaban. Y las nuevas formaciones ya no tenían las características políticas clásicas europeas.

No obstante, hay que tratar con cuidado las posibles analogías de la esfera política, como lo hizo Zoltán Kollár, quien en su último libro estudió las posibles analogías entre América Latina y Europa Central-Oriental, comparando su estructura económica y su situación periférica en la economía mundial ${ }^{22}$.

${ }^{27}$ Ibidem, 863-891.

${ }^{28}$ Ádám ANDERLE, "Latin-Amerikai utakon”, in: Népsqabadság, 1/6/1991. Melléklet, 1.

29 Zoltán KOLLÁR, Dél Keresztje alatt. Latin-Amerika latinamerikanizálódása, Budapest, 1996. Un análisis temprano fue hecho por Béla KÁDÁR, Latin-Amerika gazdasági dilemmái, Budapest, Kossuth, 1977. 
Kollár trata como posibles e iguales los siguientes criterios: el estatismo fuerte, las tendencias monopolísticas presentes en la economía dual, los intentos de la modernización iniciados desde arriba, el endeudamiento y los desequilibrios, la dependencia exterior intensiva y el mercado interior subdesarrollado y desintegrado ${ }^{30}$.

$Y$ añade: "en nuestra área también puede desarrollarse un conjunto de fenómenos de carácter desfavorable que entra dentro de la latinoamericanización." Sin embargo, Zoltán Kollár hace un inciso importante: "Todo esto se debe más probablemente a nuestra propia tendencia de desarrollo. Por lo tanto, ¿no sería más conveniente llamar a estos fenómenos indeseados como 'europa-orientalización?'?31 - pregunta.

Kollár, por lo tanto, retoma la pregunta de Wittman y Zsigmond Pál Pach: estas dos regiones se conectan a las regiones centrales desarrolladas como periferias desde la primera etapa de la formación de la economía mundial. $\mathrm{Y}$ como el pensamiento húngaro lo ha descrito varias veces: el desarrollo húngaro osciló y oscila entre Occidente y Oriente; ahora está "europaorientalizándose".

$\mathrm{Y}$ añadimos que la nivelación económica y la modernización de algunas regiones de América Latina parecen ser más perceptibles.

Es por esto que Zoltán Kollár examina los trabajos de los pensadores económicos latinoamericanos ${ }^{32}$, y sugiere el estudio del desarrollo/fomento latinoamericano.

En resumen, el pensamiento sobre el balance de la guerra de independencia y de los dos siglos de la independencia misma puede ser un llamamiento al análisis comparativo y a la extracción de conclusiones respecto a la situación histórico-económica mundial común".

30 Ibid. 87-91.

31 Ibid. 91.

32 Vid. Zoltán KOLLAiR, Fejlódés válságban. Tanulmányok Latin-Amerika gazdaságíńl. Szeged, JATE, 1988; Id. Stabilizáció vagy modernizáció. A latin-amerikai gazdaság az exredforduli elött (1960-1990). Budapest, Budapesti Közgazdaságtudományi Egyetem, 1992. 